\title{
Structures and energetics of silicon nanotubes from molecular dynamics and density functional theory
}

\author{
Amritanshu Palaria, ${ }^{1}$ Gerhard Klimeck, ${ }^{1}$ and Alejandro Strachan ${ }^{2, *}$ \\ ${ }^{1}$ School of Electrical and Computer Engineering, Purdue University, West Lafayette, Indiana 47907, USA \\ ${ }^{2}$ School of Materials Engineering, Purdue University, West Lafayette, Indiana 47907, USA
}

(Received 1 September 2008; published 13 November 2008)

\begin{abstract}
We use molecular dynamics with a first-principles-based force field and density functional theory to predict the atomic structure, energetics, and elastic properties of Si nanotubes. We find various low-energy and low-symmetry hollow structures with external diameters of about $1 \mathrm{~nm}$. These are the most stable structures in this small-diameter regime reported so far and exhibit properties very different from the bulk. While the cohesive energies of the four most stable nanotubes reported here are similar (from 0.638 to $0.697 \mathrm{eV}$ above bulk Si), they have disparate Young's moduli (from 72 to $123 \mathrm{GPa}$ ).
\end{abstract}

DOI: 10.1103/PhysRevB.78.205315

PACS number(s): 61.46.Np, 62.25.-g, 64.70.Nd

\section{INTRODUCTION}

One-dimensional (1D) nanostructures [nanotubes (NTs) and nanowires (NWs)] are expected to play a key role in a wide variety of applications ranging from electronics and optoelectronics to energy conversion. ${ }^{1,2}$ These materials not only have the potential to make bottom-up device fabrication and continued miniaturization possible, but also exhibit behavior and properties very different from their macroscopic counterparts due to size-dependent microstructure or nanostructure, quantum confinement, and even changes in crystal structure and atomic bonding. Thus, knowledge of the atomic-level structure of nanoscale materials is critical to understand and, eventually, control and optimize their properties and performance. Unfortunately, both computational prediction and experimental characterization of the atomic structure of NTs and NWs remain as challenging problems.

Several researchers have studied structures of Si NWs and NTs. For diameters over about $5 \mathrm{~nm}$, SiNWs often consist of diamond cores surrounded by an oxide layer. ${ }^{3}$ Onedimensional $\mathrm{Si}$ structures with diameter approaching $1 \mathrm{~nm}$ have recently been achieved, and significant structural uncertainties remain in this small-diameter regime that includes oxide-free NWs (Ref. 4) and hollow NTs. ${ }^{5,6}$ The several hollow and nonhollow structures proposed and theoretically characterized in recent years include fullerene-based structures ${ }^{5}$ and hexagonal wires, ${ }^{7}$ polycrystals with fivefold symmetry, ${ }^{8}$ carbon NT structure, ${ }^{9}$ and square, pentagonal, and hexagonal single-walled NTs. ${ }^{10}$ These structures were proposed based on intuition or the behavior of similar materials, and some are high-energy configurations unlikely to be observed experimentally. To address this limitation, we propose a computational approach to predict the atomic configuration of nanostructures and apply it to Si NTs; we find various hollow, low-energy structures with similar stability but disparate Young's moduli.

\section{COMPUTATIONAL APPROACH AND DETAILS}

Our approach to predict the structure and properties of nanoscale materials consists of two steps: (i) starting from a simple trial structure we use molecular dynamics (MD) with an accurate force field to carry out an annealing procedure, which explores configuration space and leads to well-relaxed structures, (ii) the most promising structures obtained from the MD simulations are fully relaxed using energy minimization with DFT within the generalized gradient approximation (GGA). Thus, our strategy combines a computationally less intensive approach to explore configuration space with a more accurate and computationally intensive method to refine the results. We emphasize that this is a generally applicable approach that can be used with any material for which an accurate force field exists. The force field should be able to capture how environment affects bonding and describe configurations very different from the bulk ground state; this is critical since the DFT minimization [step (ii)] will lead to the local minimum directly downhill from the initial configuration.

For our MD simulations we use a first-principles-based reactive force field denoted $\mathrm{ReaxFF},{ }^{11}$ a description based on the concept of partial bond orders that has been parametrized using $a b$ initio data. ReaxFF has been shown to accurately describe atomic interactions in $\mathrm{Si}$, including the process of bond breaking and formation as well as crack propagation. ${ }^{12}$ The DFT calculations are performed using GGA (Ref. 13) using the code SEQQUEST. ${ }^{14}$ SEQQUEST uses local orbital basis sets of atom- and potential-dependent contracted Gaussian functions and nonlocal norm-conserving Hamman pseudopotential developed for the GGA functional. All our NT simulations employ two $k$ points in the periodic direction (along the axis of the wire) for reciprocal space calculations. In order to confirm our DFT predictions we also used ABINIT, ${ }^{15}$ a DFT code that uses plane-wave basis sets. For ABINIT simulations, we used a kinetic-energy cutoff of 12 hartree, Troullier-Martins norm-conserving pseudopotentials and the same number of $k$ points used in the SEQQUEST calculations.

\section{RESULTS AND DISCUSSION}

\section{A. MD-DFT annealing procedure}

We use pentagonal and hexagonal NTs (rows of atoms arranged in pentagons or hexagons) as our trial structures 

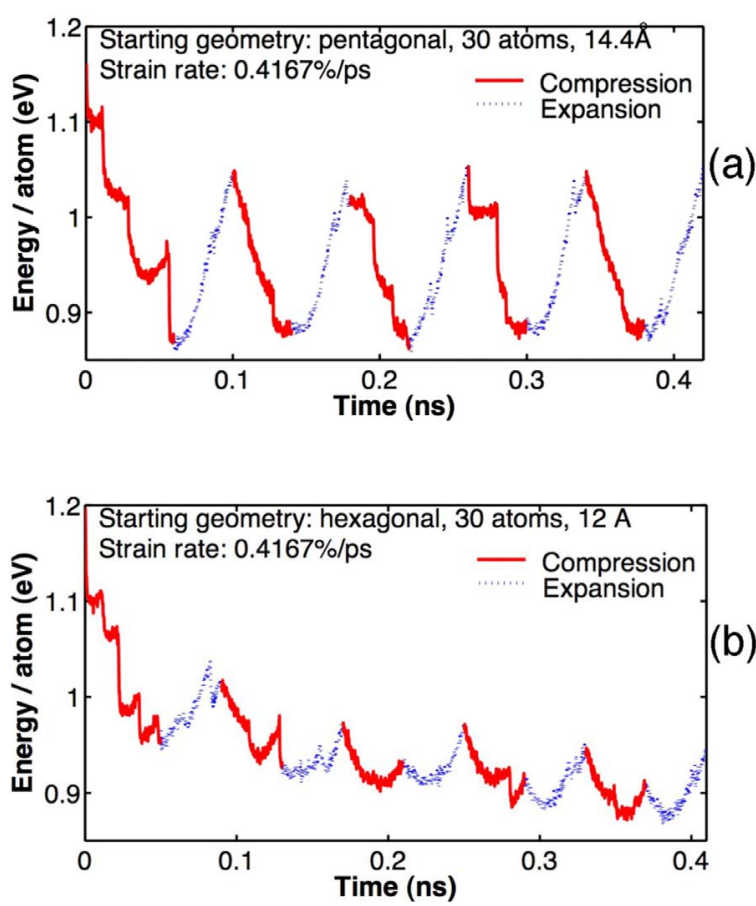

FIG. 1. (Color online) Energy as a function of time during cyclic loading with ReaxFF at $300 \mathrm{~K}$ of (a) an initially pentagonal tube of 30 atoms strained by $0.06 \AA$ /ps, and (b) an initially hexagonal tube of 30 atoms strained by $0.05 \AA$ / ps. The solid curves represent compression, and the dotted part represents expansion of the tube.

since they are the simplest metastable tubular structures. ${ }^{10}$ Different trial geometries with 5, 6, 10, 11, and 12 rows of pentagons or hexagons allow for different possible periodicities of the final structure. To obtain well-relaxed NTs, the trial structures are cyclically compressed and expanded using $\mathrm{MD}$ at two temperatures (300 and $600 \mathrm{~K}$ ). For each initial configuration and temperature, we apply various cyclic mechanical loadings: three ranges of strain $(-37.50 \%<\varepsilon<$ $-12.50 \%, \quad-25.00 \%<\varepsilon<-8.30 \%$, and $-20.87 \%<\varepsilon<$ $-4.17 \%)$ and three strain rates $(0.04167,0.41667$, and $0.83333 \% / \mathrm{ps}$ ). This makes thermal and mechanical energy available to the system to overcome energy barriers that may separate the initial trial structure from lower-energy configurations and allows the NT to find its natural length. Figure 1(a) shows the potential energy as a function of time for an MD annealing simulation of a pentagonal initial structure at $T=300 \mathrm{~K}$ and strain rate of $0.4167 \% / \mathrm{ps}$. It shows abrupt energy drops that correspond to configurational changes and rather well-behaved energy-length relationships between structural transitions. In this example, the lowest-energy configuration is attained in the first-compression-expansion cycle, and subsequent mechanical cycles do not result in additional relaxation. But there are other cases, see Fig. 1(b), where several cycles are required before a periodic behavior is achieved. The various strain rates and deformation ranges result in simulation time scales ranging from 0.2 to $2.7 \mathrm{~ns}$. (Note that this is well beyond what is possible today with $a b$ initio MD.)

An analysis of the structures resulting from the MD annealing procedure leads to the following general observa-

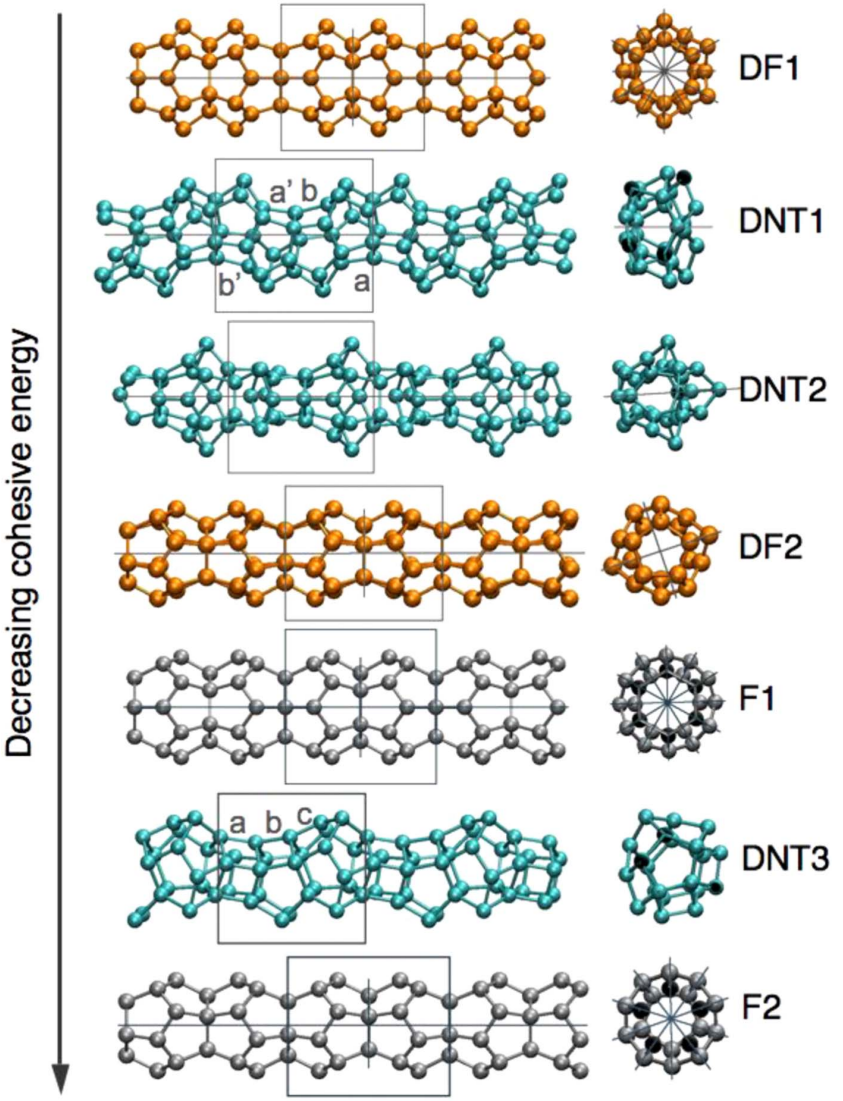

FIG. 2. (Color online) Hollow Si nanotube structures, arranged in the order of increasing cohesive energy. DNT1, 2, and 3 are obtained from the MD-DFT method and DF1 and DF2 from DFT minimization. The solid boxes in the longitudinal view mark the boundaries of a unit cell. The lines in the longitudinal and crosssectional views represent the different axes and planes of symmetry referred to in the text. As explained in the text, DF1 and DF2 are topologically equivalent to F1 and F2 but have lower symmetry.

tions: (i) the initial structures consisting of 11 and 12 rows of atoms buckle and do not lead to one-dimensional structures for the high strain rates accessible to MD; (ii) about $15 \%$ of the simulations starting from 5,6 , or 10 row structures lead to hollow 1D tubes; (iii) pentagonal trial structures lead to a larger percent of nanotubes as compared to hexagonal ones; (iv) on average, lowering the strain rate results in a larger percent of hollow 1D structures; the highest rate we studied did not result in any tubular structure.

We selected the eight most promising configurations from the MD simulations and relaxed them using the DFT code SEQQUEST. DFT relaxation of the MD structures led to lowsymmetry disordered tubular structures with similar energetics (within a range of $0.05 \mathrm{eV} /$ atom); the eight structures converged into three topologically distinct NTs during minimization. These final three structures were relaxed using the plane-wave code ABINIT to confirm our results.

\section{B. Energetics of Si nanotubes}

Figure 2 shows the atomic structures of the lowest-energy Si NTs known to date with diameters around $1 \mathrm{~nm}$. As will 
TABLE I. DFT-GGA properties of silicon nanostructures. DNT1, DNT2, and DNT3 are disordered structures obtained from our annealing procedure; DF1 and DF2 are distorted fullerene structures, and F1 and F2 are the corresponding parent structures. Simple pentagonal (Pen), hexagonal (Hex), and carbon nanotube (CNT) structures are also shown. We also show two simple-hexagonal wires with different radii; these nonhollow structures are denoted SHW1 and SHW2. For each structure, we report its periodic length $(L)$, number of atoms in its unit cell $\left(N_{\mathrm{at}}\right)$, cross-sectional area $(A)$, energy (per atom and with respect to diamond structure) obtained from SEQQUeST $\left(\varepsilon_{S}\right)$, Young's modulus ( $E$, from SEQQUEST), and energy obtained from SEQQUEST $\left(\varepsilon_{A}\right)$. Nanotube structures F1, F2, Pen, Hex, and CNT $(4,4)$ as well as the nonhollow structures SHW1 and SHW2 have been reported previously and are included for comparison.

\begin{tabular}{lrrrccc}
\hline \hline Structure & \multicolumn{1}{c}{$\begin{array}{c}\text { L } \\
\AA\end{array}$} & \multicolumn{1}{c}{$N_{\text {at }}$} & \multicolumn{1}{c}{\begin{tabular}{c}
\multicolumn{1}{c}{$\left(\AA^{2}\right)$} \\
DF1
\end{tabular}} & $\begin{array}{c}\varepsilon_{\mathrm{S}} \\
(\mathrm{eV})\end{array}$ & $\begin{array}{c}E \\
(\mathrm{GPa})\end{array}$ & $\begin{array}{c}\varepsilon_{\mathrm{A}} \\
(\mathrm{eV})\end{array}$ \\
\hline DNT1 & 10.64 & 36 & 80.9 & 0.638 & 118 & 0.605 \\
DNT2 & 10.58 & 30 & 86.0 & 0.673 & 86 & 0.625 \\
DF2 & 10.93 & 30 & 67.6 & 0.697 & 123 & \\
F1 & 10.86 & 36 & 72.2 & 0.705 & 145 & \\
DNT3 & 9.79 & 25 & 69.4 & 0.708 & 50 & 0.653 \\
F2 & 10.97 & 30 & 57.9 & 0.714 & 179 & 0.668 \\
Pen & 2.42 & 5 & 33.3 & 0.755 & 273 & \\
Hex & 2.40 & 6 & 40.7 & 0.774 & 493 & \\
CNT $(4,4)$ & 3.89 & 16 & 324.7 & 0.805 & 53 & \\
SHW1 & 2.49 & 13 & 90.2 & 0.663 & 305 & \\
SHW2 & 2.64 & 7 & 40.7 & 0.751 & 140 & \\
\hline \hline
\end{tabular}

be described in detail below, these structures were obtained either by our MD-DFT approach or by energy minimization using DFT; the fullerene-based structures F1 and F2 have been reported before and are included for comparison. As will be described shortly, two of the five new structures are topologically equivalent to F1 and F2 but have lower symmetry. The disordered nanotube (DNT) structures resulting from our MD-DFT approach are denoted DNT1, DNT2, and DNT3; these were obtained from 30-atom pentagonal, 30atom hexagonal, and 25-atom pentagonal trial structures, respectively. Together with the DNTs, we show fullerene-based structures that distort if their full symmetry (discussed in Sec. III C) is not enforced during DFT energy minimization and relaxation; these low-energy low-symmetry distorted fullerene structures are denoted DF1 and DF2. It is interesting to note that structure DNT2, resulting from our annealing procedure, is a defective form of fullerene structure DF2 with lower energy than its parent structure. The atomic structures of DNTs and DFs are provided in the supplementary material. ${ }^{16}$ Structures F1 and F2 in Fig. 2 are fullerene-based and were proposed earlier. ${ }^{5}$ For comparison, we also simulated using DFT-GGA in SEQQUEST other Si nanostructures proposed earlier. Table I summarizes properties of all the structures simulated by us (all energies are given per atom and with respect to the diamond structure). The table shows various low-energy tubular structures with similar stability; these structures are either DNTs obtained from our MD-DFT procedure or fullerenelike. Table I also shows that the pentagonal and hexagonal trial structures (denoted Pen and Hex) are highly strained high-energy structures, and so are carbon nanotube-based structures. We find $0.805 \mathrm{eV}$ of energy for the armchair $(4,4)$ carbon nanotube $(\mathrm{CNT})$ structure. [Zhang et al. ${ }^{9}$ found armchair configurations to be more favorable than zigzag $(m, 0)$ structures.]

To facilitate comparisons between the various structures, we also report in Table I their effective cross-sectional areas. We define this area as that of the circumcylinder that encloses all atoms in the structure plus roughly a half $\mathrm{Si}-\mathrm{Si}$ bond distance $(1.20 \AA)$ along its periphery; this is a conservative measure, and in some cases, e.g., DNT1 and DNT2, it encloses considerable extra area external to the tube (in addition to inner area). With this definition, the predicted structures range in diameter from 9 to $11 \AA$ and are comparable in energy to wires (nonhollow structures) of similar size. Table I includes properties of simple-hexagonal wires of different radii (SHW1 and SHW2); these structures were reported to be among the lowest-energy 1D structures in the small-diameter regime by Kagimura et al. ${ }^{7}$ The two lowestenergy nanotubes (DF1 and DNT1) are more stable than the hexagonal wire of similar diameter. We also used DFT-GGA to calculate the Young's moduli of the structures by performing a series of structural relaxation at various levels of strain in the range $-0.02<\varepsilon<0.02$. Young's moduli of the seven most stable tubular structures vary by over a factor of three between 50 and $179 \mathrm{GPa}$ (bulk Si is $150 \mathrm{GPa}$ ). We confirmed the relative energetics of the new structures by repeating structural relaxations using the plane-wave DFT software ABINIT (see last column of Table I). ABINIT leads to slightly lower energy (always with respect to the corresponding description of bulk diamond) and conforms the relative stability of the various structures.

\section{Atomic-level structures of Si nanotubes}

The fullerene-based structures F1 and F2 have sixfold and fivefold rotational symmetries around the tube axis, respectively. The other rotational axes (twofold) and reflection planes are shown by lines in the cross-sectional view of the structures in Fig. 2. The symmetry operations for F1 are $\left\{(E \mid \boldsymbol{t}),\left(C_{12} \mid \boldsymbol{t} / 2\right), D_{6 h}\right\}$, and for F2 they are $\left\{(E \mid \boldsymbol{t}),\left(C_{10} \mid \boldsymbol{t} / 2\right), D_{5 h}\right\} . E$ is the identity operation, $\boldsymbol{t}$ represents the translational periodicity of the simulation cell along its axis, $\left(C_{12} \mid \boldsymbol{t} / 2\right)$ and $\left(C_{10} \mid \boldsymbol{t} / 2\right)$ represent screw symmetry operations, and finally $D_{6 h}$ and $D_{5 h}$ are the Schönflies representation of the point group symmetries. Structure DF2 exhibits a considerable loss of symmetry compared to its parent structure F2 with only $\left\{(E \mid \boldsymbol{t}),\left(C_{2} \mid \boldsymbol{t} / 2\right),\left(\sigma_{v} \mid \boldsymbol{t} / 2\right), D_{1 h}, \sigma_{v}^{\prime}\right\}$ allowed; $\left(\sigma_{v} \mid t / 2\right)$ denotes glide reflection on a vertical plane containing the principal axis, and $\sigma_{v}^{\prime}$ represents reflection through another vertical plane. Ponomareva et al. ${ }^{17}$ reported another distorted form of F2 with symmetry operations $\left\{(E \mid t), D_{5 h}\right\}$ (but its atomic structure was not specified in detail). Similarly, DF1 with $\left\{(E \mid \boldsymbol{t}), D_{6 h}\right\}$ has lower symmetry than F1. The MD annealing procedure substantially reduces both the translational and rotational symmetries of the initial 
trial structures, and the resulting DNT structures have less symmetry than fullerene-based ones. The translational symmetry of all DNTs along their axes is reduced to the minimum allowed by the periodic boundary conditions. All DNTs can be described as a chain of atomic cages interconnected by five- or six-membered rings. DNT1 consists of four cages, denoted $a, b, a^{\prime}$, and $b^{\prime}$ within its unit cell (see Fig. 2). The cages are connected by five-atom ring cross sections, and $a^{\prime}$ and $b^{\prime}$ are reflections of $a$ and $b$, respectively, through a plane $\sigma_{v}$, parallel to the longitudinal axis of the tube and marked by lines in the two views of the structure in Fig. 2. The space symmetry of DNT1 is $\left\{\{E \mid \boldsymbol{t}\},\left(\sigma_{v} \mid \boldsymbol{t} / 2\right)\right\}$. Structure DNT2 is a defective form of the 30-atom-unit-cell fullerene structure DF2 in which an atom from one of the two cages moves into the other one causing significant geometrical distortions. The symmetry operations of DNT2 are $\left\{(E \mid t), C_{1 v}\right\}$ (the mirror plane is shown in Fig. 2). Finally, three cages connected by five-membered atomic rings can describe structure DNT3 with symmetry $(E \mid \boldsymbol{t})$. We found two enantiomers of this structure when performing MD simulations. One was found when straining the 25-atom pentagonal structure within $-37.50 \%<\varepsilon<-12.50 \%$ with $\varepsilon=0.04167 \% /$ ps and the other when straining it within $-25.00 \%<\varepsilon<-8.30 \%$ at ten times the rate. This indicates that our approach is robust, and the resulting structures can be generated by various pathways.

Additional insight into the various structures can be gained from radial distribution functions (RDFs), shown in Fig. 3(a), and the distribution of coordination numbers (number of nearest neighbors), shown in Fig. 3(b). Fullerenebased structures exhibit a split first RDF peak that originates from atoms having three or four nearest neighbors, leading to three types of bonds in order of increasing length: (i) between two three-coordinated atoms, (ii) between a threecoordinated and a four-coordinated atom and, (iii) between two atoms with four nearest neighbors. [Only two peaks are seen in Fig. 3(a) due to the resolution of the RDF calculation.] On the other hand, the disordered nanotube structures exhibit a single first peak since the atomic bonding environment cannot be grouped into two well-defined categories. A second difference in the RDFs is that the second peak for the DNTs is significantly more diffuse than that for the fullerene ones. Angle distribution functions of the DNTs, see Fig. 3(b), are also significantly more diffuse than those of fullerene nanotubes, a further indication of the disorder of the structures. Figure 3(c) shows the distribution of coordination numbers for the various Si NT structures; in order to define first nearest neighbors we use a cutoff distance of $2.70 \AA$ (15\% larger than the Si-Si bond distance in diamond). Figure 3(c) shows that fullerene structures have twice as many three-bonded atoms than four-bonded ones. This ratio gets modified in favor of the higher coordination for the DNTs that contain more four-coordinated atoms than threecoordinated ones; furthermore, the histograms also show some five-coordinated atoms. This last number is very sensitive to the choice of cutoff as is commonly found in disordered structures and apparent from the RDF plots. For instance, if the cutoff is reduced to $2.50 \AA$, no "five-bonded" atoms are found.
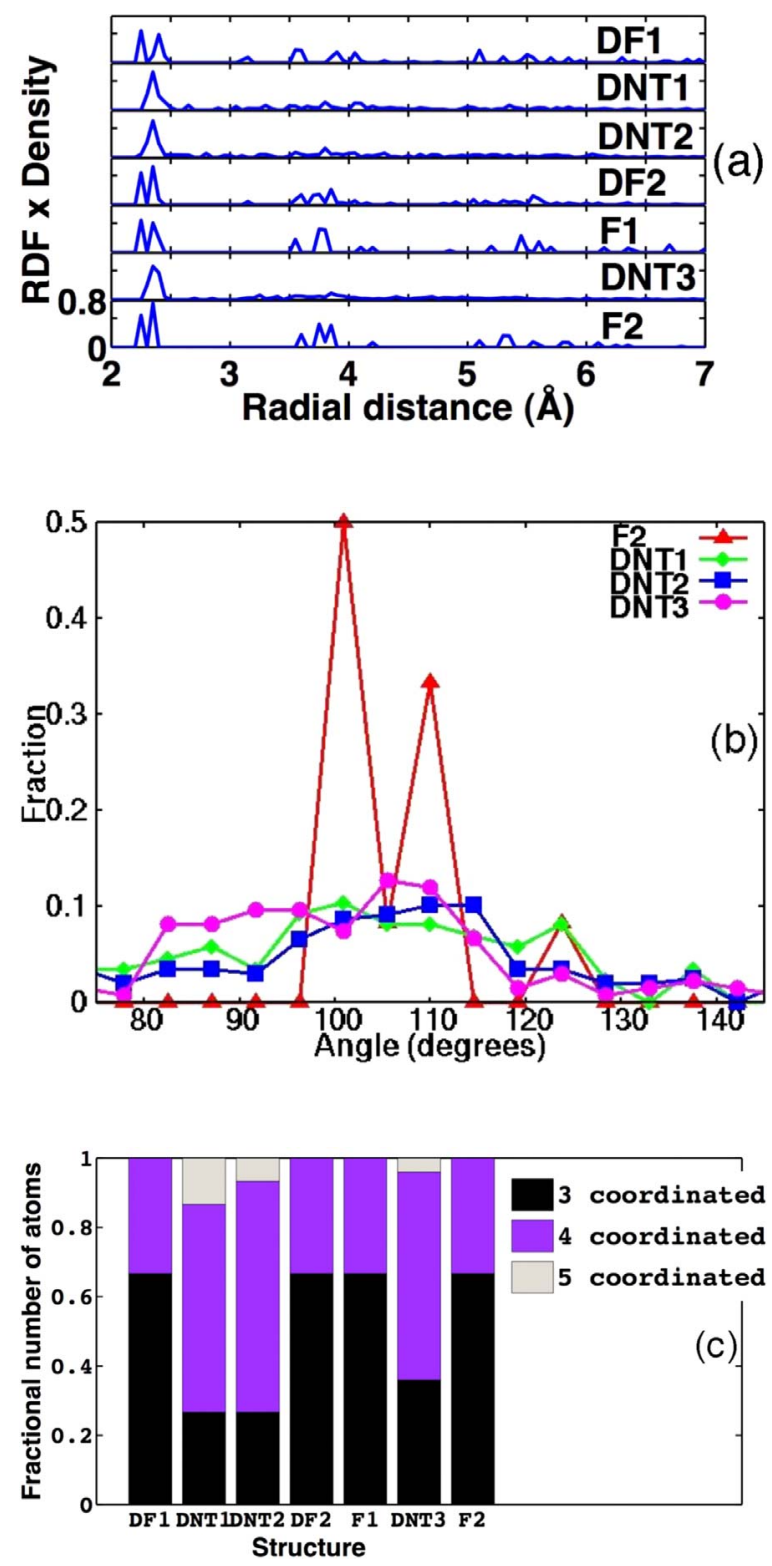

FIG. 3. (Color online) (a) The atomic density times radial distribution function plot for various $\mathrm{Si}$ nanotube structures, obtained at a resolution of $0.05 \AA$. (b) Bond angle distribution for a fullerene SiNT (F2) and the three disordered nanotubes (DNTs). (c) Histogram showing the fractional number of 3,4 , and 5 coordinated atoms in the nanotube structures.

\section{CONCLUSIONS}

In this paper we present a generally applicable procedure for the prediction of the atomic structure of nanoscale materials and apply it to Si NTs. We find various low-energy structures with similar stability (within $0.07 \mathrm{eV} /$ atom of one another) but with widely different atomic structures and disparate elastic properties (Young's moduli within a factor of about 3 ). These structures exhibit low symmetry and bonding environments significantly different from those known for bulk silicon. The predicted hollow structures are the most stable one-dimensional structures known for $\mathrm{Si}$ in the 1-nm diameter range and may be found experimentally under ultrahigh vacuum conditions. 


\section{ACKNOWLEDGMENTS}

A.S. would like to thank A. C. T. van Duin and W. A. Goddard, III for the use of ReaxFF code and P. A. Schultz for help with SEQQUEST. This work was partially supported by the Semiconductor Research Corporation, the Microelectron- ics Advanced Research Corporation and its Focus Center on Materials, Structures and Devices, by the Network for Computational Nanotechnology through nanoHUB.org computational resources funded by the U.S. National Science Foundation under Grant No. EEC-0228390, and by Purdue University.
*Author to whom correspondence should be addressed. strachan@purdue.edu

${ }^{1}$ J. Xiang, W. Lu, Y. Hu, Y. Wu, H. Yan, and C. M. Leiber, Nature (London) 441, 489 (2006).

${ }^{2}$ A. I. Hochbaum, R. Chen, R. D. Delgado, W. Liang, E. C. Garnett, M. Najarian, A. Majumdar, and P. Yang, Nature (London) 451, 163 (2008).

${ }^{3}$ A. M. Morales and C. M. Leiber, Science 279, 208 (1998).

${ }^{4}$ D. D. D. Ma, C. S. Lee, F. C. K. Au, S. Y. Tong, and S. T. Lee, Science 299, 1874 (2003).

${ }^{5}$ B. Marsen and K. Sattler, Phys. Rev. B 60, 11593 (1999).

${ }^{6}$ M. De Crescenzi, P. Castrucci, M. Scarselli, M. Diociaiuti, P. S. Chaudhari, C. Balasubramanian, T. M. Bhave, and S. V. Bhoraskar, Appl. Phys. Lett. 86, 231901 (2005).

${ }^{7}$ R. Kagimura, R. W. Nunes, and H. Chacham, Phys. Rev. Lett. 95, 115502 (2005).

${ }^{8}$ Y. Zhao and B. I. Yakobson, Phys. Rev. Lett. 91, 035501 (2003).

${ }^{9}$ M. Zhang, Y. H. Kan, Q. J. Zang, Z. M. Su, and R. S. Wang, Chem. Phys. Lett. 379, 81 (2003).
${ }^{10}$ J. Bai, X. C. Zeng, H. Tanaka, and J. Y. Zeng, Proc. Natl. Acad. Sci. U.S.A. 101, 2664 (2004).

${ }^{11}$ A. C. T. van Duin, A. Strachan, S. Stewman, Q. Zhang, X. Xu, and W. A. Goddard III, J. Phys. Chem. A 107, 3803 (2003).

${ }^{12}$ M. J. Buehler, A. C. T. van Duin, and W. A. Goddard, Phys. Rev. Lett. 96, 095505 (2006).

${ }^{13}$ J. P. Perdew, K. Burke, and M. Ernzerhof, Phys. Rev. Lett. 77, 3865 (1996).

${ }^{14}$ P. A. Schultz, SEQQUEST Project (http://dft.sandia.gov/Quest/).

${ }^{15}$ The ABINIT code is a common project of the Université Catholique de Louvain, Corning Incorporated, and other contributors (http://www.abinit.org).

${ }^{16}$ See EPAPS Document No. E-PRBMDO-78-010844 for the structure geometry data (xyz files) of the SiNTs depicted in Fig. 2. For more information on EPAPS, see http://www.aip.org/ pubservs/epaps.html.

${ }^{17}$ I. Ponomareva, M. Menon, D. Srivastava, and A. N. Andriotis, Phys. Rev. Lett. 95, 265502 (2005). 